\title{
A REMARK ON FINITE DIMENSIONAL COMPACT CONNECTED MONOIDS
}

\author{
L. W. ANDERSON* AND R. P. HUNTER ${ }^{1}$
}

ABSTRACT. Let $S$ be a compact $n$-dimensional monoid. Let $A$ be a compact connected subsemigroup algebraically irreducible from the minimal ideal to the identity of $S$. Then there exists a closed proper ideal $J$ such that $\operatorname{dim}\{A \mid A \cap J\} \leqq \operatorname{dim} S-\operatorname{dim} H_{1}$.

If $S$ is as above, then, according to a result of Wallace [6], the dimension of the maximal subgroup at the identity $H_{1}$ cannot exceed $\operatorname{dim} S-1$. In fact, if $\operatorname{dim} H_{1}=\operatorname{dim} S-1$, then there is a local thread at the identity meeting $H_{1}$ at $\{1\}$ [5]. This is a corollary of the following

PROPOSITION 1. Let $S$ be a compact connected semigroup, with identity 1 . Let $A$ be a compact connected subsemigroup algebraically irreducible from $\{1\}$ to the minimal ideal of $S$. Then there exists a closed proper ideal J such that

$$
\operatorname{dim}\{A \mid A \cap J\} \leqq \operatorname{dim} S-\operatorname{dim} H_{1}
$$

In the above, dimension is taken in the sense of Cohen [3]. The canonical reference throughout is [5].

It seems not unreasonable to conjecture that if $\operatorname{dim} A=\operatorname{dim} S-\operatorname{dim} H_{1}$, notations as above, then some form of uniqueness might be established for $A$. We include the following example to show that this is, unfortunately, not the case. Indeed we have the following

PROPOSITION 2. For any $n>0$ there is a compact connected abelian semigroup $S$ of dimension $n$ with identity 1 and zero 0 containing two algebraically irreducible subsemigroups $A_{1}$ and $A_{2}$ such that $A_{1} \cap A_{2}=\{0,1\}$ such that, given any closed proper ideal $J$,

$$
\operatorname{dim} A_{1} / A_{1} \cap J=\operatorname{dim} S=\operatorname{dim} A_{2} / A_{2} \cap J .
$$

The proof of Proposition 1 will be broken down into a few lemmas some of which may be of interest to the reader.

Received by the editors February 20, 1973.

AMS (MOS) subject classifications (1970). Primary 22A15, 22C05, $22 \mathrm{~F} 45$.

Key words and phrases. Semigroup, algebraically irreducible, dimension.

* Posthumous

${ }^{1}$ I should like to thank the referee for several helpful remarks and in particular for the drawings for Figures 1 and 2.

(C) American Mathematical Society 1974 
LEMMA 1. Let $S$ be a compact monoid with $A$ a compact abelian subsemigroup such that $A \cap H_{1}=\{1\}$ and $E(A)$ ordered by $e \leqq f \Rightarrow e f=f e=e$. For $e^{2}=e \in A$ let $B_{e}$ denote the maximal subgroup of $A$ determined by $e$. Let

$$
Y_{e}=\left\{g \mid g \in H_{1}, g e \in B_{e}\right\}=\left\{g \mid g \in H_{1}, g B_{e}=B_{e}\right\} .
$$

Then $Y_{e}$ is a closed subgroup of $H_{1}$. If $H_{1}$ has finite dimension there is a closed proper ideal $J$ such that $e^{2}=e \notin A \cap J$ implies $\operatorname{dim} Y_{e}=0$. If $H_{1}$ is connected, as well as finite dimensional, then $J$ may be chosen so that $Y_{e}$ is both zero dimensional and central in $H_{1}$.

Proof. Since the idempotents of $A$ are ordered and $A$ is abelian, the collection of subgroups $\left\{Y_{e}\right\}$ of $H_{1}$ is ordered by inclusion. Thus, $e \leqq f$ implies $Y_{e} \supseteq Y_{f}$. Clearly we may, without loss of generality, assume that there are idempotents of $A$, below and arbitrarily close to 1 . Since $A \cap H_{1}=$ $\{1\}$, it follows from continuity of multiplication that the common part of the groups $\left\{Y_{e}\right\}, e \neq 1, e \in A$, is precisely $\{1\}$.

Since a compact connected group cannot contain a proper compact connected subgroup of the same finite dimension, it follows that, for some $f^{2}=f$, the corresponding $Y_{f}$ is zero dimensional. Certainly the ideal generated by $f$ can be taken as $J$.

The last claim of the lemma follows from the following fact: If $G$ is a compact connected finite dimensional group and $\left\{G_{\alpha}\right\}$ is a collection of closed subgroups ordered by inclusion with intersection equal to the identity of $G$ then there is some $\alpha$ such that $G_{\alpha}$ is zero dimensional and central.

Indeed, $G$ can be given as $L \times C / F$ where $L$ is semisimple, $C$ is connected and abelian and $F$ is central, finite and such that $F \cap C=\{1\}$. If $q$ is the natural homomorphism of $L \times C$ onto $G$ then the collection $\left\{q^{-1}\left(G_{\alpha}\right)\right\}$ is ordered by inclusion and $\left\{\pi\left(q^{-1}\left(G_{\alpha}\right)\right)\right\}$ is a nested collection where $\pi: L \times C \rightarrow L$ is the projection onto $L$.

Since $L$ is a compact Lie group, the collection $\pi\left(q^{-1}\left(G_{\alpha}\right)\right)$ can have only finitely many distinct members. Thus for some $\alpha$ we have that $\pi\left(q^{-1}\left(G_{\alpha}\right)\right)$ is trivial so $q^{-1}\left(G_{\alpha}\right) \subset C$ so that $G_{\alpha} \subset q(C)=Z(G)$.

LEMMA 2. If $S$ is a compact monoid and $B$ a compact subgroup with identity $e$, then the product $H_{1} B$ is homeomorphic to a homogeneous space $H_{1} \times B / \tilde{Y}$ where $\tilde{Y}$ is a subgroup of $H_{1} \times B$ isomorphic with

$$
Y_{e}(B)=Y_{e}=\left\{g \mid g \in H_{1}, g e \in B\right\}=\left\{g \mid g \in H_{1}, g B=B\right\} .
$$

Thus, if $Y_{e}$ is central in $H_{1}$ and $H_{1} e \cap B$ lies in the center of $B$, then $H_{1} B$ is the underlying space of a compact group. 
Proof. Consider the action of $H_{1} \times B$ on Se defined by $((g, b), x) \rightarrow$ $g x b^{-1}$. The orbit at $e$ is $H_{1} e B=H_{1} B$ and the stability group $\tilde{Y}$ at $e$ is

$$
\left\{(g, b) \mid g e b^{-1}=e\right\}=\{(g, b) \mid g e=b\}=\left\{\left(g,(g e)^{-1}\right) \mid g \in Y_{e}\right\} .
$$

Now $\tilde{Y}$ and $Y_{e}$ are isomorphic under the correspondence $(g, b) \leftrightarrow g$. Clearly if $g$ lies in the center of $H_{1}$ and $g e=b$ lies in the center of $B$ then $(g, b)$ is in the center of $H_{1} \times B$.

Lemma 3. Let $S$ be a compact connected monoid of finite dimension. Let $H_{1}$ be connected and let $B$ be a compact connected abelian subgroup with identity e such that $Y_{e}(B)=Y_{e}$ is zero dimensional and central in $H_{1}$. Then $H_{1} B$ is the underlying space of a compact connected group of dimension $\operatorname{dim} H_{1}+\operatorname{dim} B$. In particular, if $H_{1} B$ does not meet the minimal ideal of $S$ then

$$
\operatorname{dim} S>\operatorname{dim} H_{1} B=\operatorname{dim} H_{1}+\operatorname{dim} B .
$$

Proof. The first claim follows from Lemma 2. If we were to have $\operatorname{dim} S=\operatorname{dim} H_{1} B$ and an element $x$ such that $x\left(H_{1} B\right) \cap H_{1} B=\square$ we would have a contradiction since we would have $H^{\operatorname{dim} S}\left(H_{1} B\right)=0$ (see [5, p. 54]). But $H_{1} B$ is topologically a compact group. With $Z$ as coefficient group we would have $H^{\text {dim } S}\left(H_{1} B\right) \neq 0$.

The following lemma follows from the same argument used in [2].

LEMma 4. Let $S$ be a compact monoid, $C$ be the component of $\{1\}$ in $H_{1}$, and $A$ be a compact connected subsemigroup algebraically irreducible from $\{1\}$ to the minimal ideal of $S$. Then $\mathrm{cl}\langle C, A\rangle$ the closure of the semigroup generated by $C$ and $A$, has $C$ for its maximal subgroup at the identity.

The semigroup $\operatorname{cl}\langle C, A\rangle$ is the compact connected semigroup algebraically irreducible about $C \cup A$.

ProOf OF PROPOSITION 1. From Lemma 4 we may assume $H_{1}$ is connected and from Lemma 3 we know that $\operatorname{dim} S>\operatorname{dim} H_{1} B_{e}$ so that

$$
\operatorname{dim} S \geqq \operatorname{dim} H_{1}+\operatorname{dim} B_{e}+1 \text {, }
$$

where $e \in E(A) \mid J$, in accordance with the second part of Lemma 1 . However, $\operatorname{dim}(A \mid A \cap J)=1+\sup \left\{\operatorname{dim} B_{e} \mid e \in E(A) \cap J\right\}$. Thus

$$
\operatorname{dim}\{A \mid A \cap J\} \leqq \operatorname{dim} S=\operatorname{dim} H_{1} .
$$

REMARK. In terms of the above notations one can infer from [4] that $\operatorname{dim} S \geqq \operatorname{dim} H_{1} B_{e}+1$. Then, using only the homogeneous structure of $H_{1} B_{e}$, one concludes that

$$
\operatorname{dim} H_{1} B_{e}=\operatorname{dim} H_{1}+\operatorname{dim} B_{e}+\operatorname{dim} Y_{e}=\operatorname{dim} H_{1}+\operatorname{dim} B_{e},
$$

via Lemma 2. 


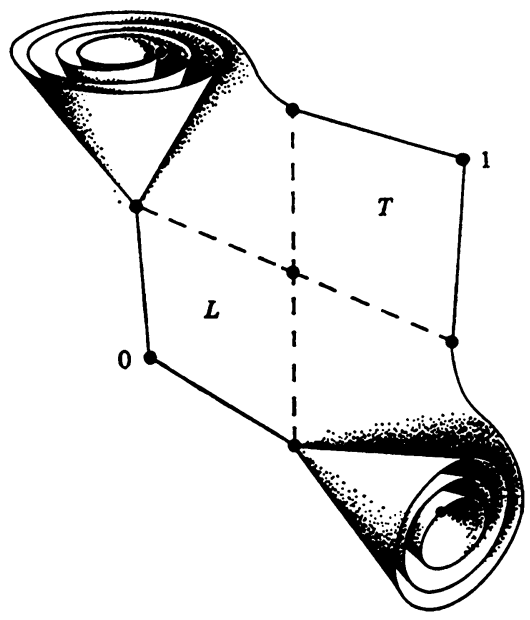

FIGURE 1

Proof of Proposition 2. Let $S$ be composed of a half-open interval winding upon a circle group $K$ so that $S / K=S / \mathscr{H}=[0, e, u]$ where $[0, e]$ and $[e, u]$ are ordinary unit intervals.

Now form $S \times S$ as usual, and decompose $S \times S$ according to $\mathscr{C}$, where the classes of $\mathscr{C}$ are sets of the form $K \times\{t\},\{t\} \in K$ or $K \times K$ where $t \in e S e$, and points otherwise. (See Figure 1.)

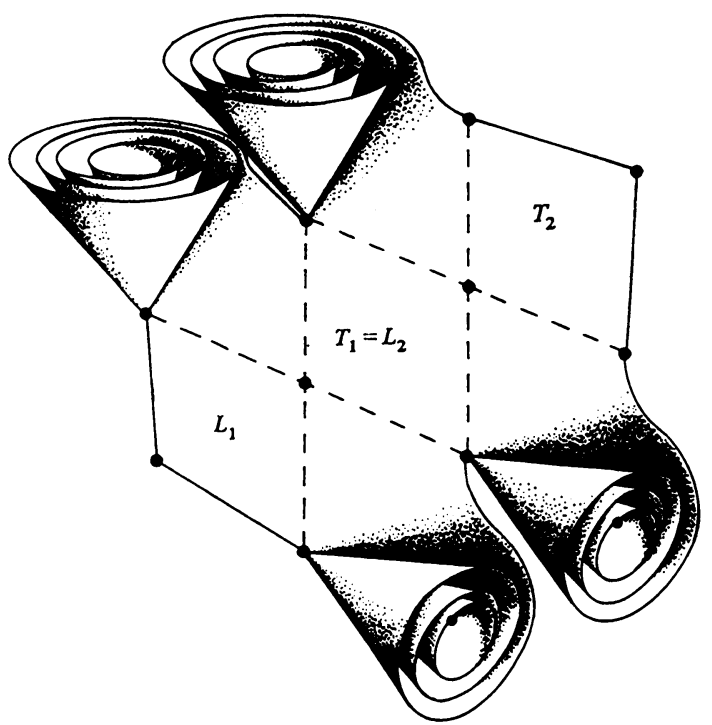

Figure 2 
This is a modified cone construction similar to those in [1]. Note $S_{1}=S \times S / \mathscr{C}$ contains two copies of $S$ meeting at the identity, and two isomorphic algebraically irreducible semigroups from zero to identity meeting at just zero and identity. Both have dimension two.

Now in terms of the figure, the ideal $L=L_{1}$ is isomorphic to the submonoid $T=T_{1}$. Both of these are isomorphic to the cartesian product of two unit intervals. Thus if $S_{2}$ is another copy of $S_{1}$ there is an isomorphism $\phi$ between $L_{2}$ and $T_{1}$, and $S_{2}$ may be attached to $S_{1}$ via $\phi$. (See Figure 2.)

The rest of the construction is now standard. The semigroups $S_{n}$ are taken converging to a point taken as identity. Clearly in place of the circle group one may use any compact connected finite dimensional abelian group.

\section{REFERENCES}

1. L. W. Anderson and R. P. Hunter, Homomorphisms and dimension, Math. Ann. 147 (1962), 248-268. MR 26 \#4324.

2. - Sur les demi-groupes compacts et connexes, Fund. Math. 56 (1964), 183-187. MR 30 \#2470.

3. H. Cohen, $A$ cohomological definition of dimension for locally compact Hausdorff spaces, Duke Math. J. 21 (1954), 209-224. MR 16, 609.

4. J. Day and K. H. Hofmann, Clan acts and codimension, Semigroup Forum 4 (1972), 206-214.

5. K. H. Hofmann and P. S. Mostert, Elements of compact semigroups, Merrill, Columbus, Ohio, 1966. MR 35 \#285.

6. A. D. Wallace, Cohomology, dimension and mobs, Summa Brazil. Math. 3 (1953), 43-55. MR 15, 336.

Department of Mathematics, Pennsylvania State University, University Park, PenNSYlVania 16802 